\title{
Avaliação da qualificação e da produção científica de docentes para formação na área de Ciências Contábeis (Accounting) em escolas de negócios de Universidades de Classe Mundial (UCM)
}

\section{Evaluation of the qualification and scientific production of teachers for training in the area of Accounting (Accounting) in business schools of World Class Universities (UCM)}

\author{
Recebimento: 17/11/2020 - Aceite: 05/01/2021 - Publicação: 01/02/2021 \\ Processo de Avaliação: Double Blind Review
}

\author{
André Yeh Lima ${ }^{1}$ \\ Graduando em Ciências Contábeis pela Universidade Federal de São Paulo (UNIFESP) \\ andrelimayeh@gmail.com
}

Heloisa Candia Hollnagel

Doutora em Ciências Biológicas pela Universidade de São Paulo (USP)

Professora do Departamento de Ciências Contábeis pela Universidade Federal de São

Paulo (UNIFESP)

heloisa.hollnagel@unifesp.br

https://orcid.org/0000-0002-5360-6271

Luiz Jurandir Simões Araújo

Doutor em Engenharia Elétrica pela Escola Politécnica (POLI USP)

Professor do Departamento de Contabilidade e Atuária da Faculdade de Economia, Administração e Contabilidade (FEA USP)

ljsimoes@usp.br

\section{RESUMO}

O docente universitário se dedica a atividades de extensão, ensino e pesquisa, esta última responsável pela expansão do conhecimento científico. Somado ao cenário de desenvolvimento constante das ciências contábeis como ciências sociais aplicadas reforça o nível de atenção que essa atividade merece. Nas chamadas Universidades de Classe Mundial (UMC), consideradas de excelência, o impacto da produção acadêmica, a captação de recursos (financiamento) e a transferência de conhecimento para a indústria são

1 Autor para correspondência: Universidade Federal de São Paulo (UNIFESP)- Rua Oleska Winogradow, $\mathrm{n}^{\circ}$ 100 - Jardim das Flores, Osasco - SP, 06110-295, Brasil 
diferenciais. Muitas organizações e empresas medem a qualidade de uma universidade por sua posição nas tabelas de classificação como o THE (Times Higher Education). A produção acadêmica de pesquisadores de UMC no exterior (2 americanas e 1 do Reino unido) foi avaliada por ferramentas cientométricas, como o Google Scholar e produção registrada na instituição. No caso da UMC brasileira, o critério foi análise do Currículo Lattes e cruzamento com o Qualis (sistema brasileiro de avaliação de periódicos). Além da produção, a titulação e o tempo de magistério foram analisados. Os dados mostram que existe influência da titulação do docente em seus índices de produção (quantidade e qualidade) e a produção de docentes com mais senioridade apresenta maior impacto. Situações semelhantes foram encontradas em Purdue, Manchester e na Universidade Federal de São Paulo UNIFESP. Os resultados de titulação e produção mostram-se distintos em Stanford, justificando sua melhor classificação no ranking.

Palavras-Chave: universidade de classe mundial, graduação em Ciências Contábeis, perfil do professor universitário, avaliação da produção científica, escolas de negócios

\section{ABSTRACT}

The professoriate faculty is dedicated to extension, teaching, and research; the latter is responsible for the expansion of scientific knowledge, which added to the scenario of the constant development of accounting sciences as applied social sciences reinforce the level of attention that should be given to this activity. In the so-called World-Class Universities $(U M C)$, considered to be of excellence, the impact of academic production, fundraising (financing) and the transfer of knowledge to the industry are differentials. Many organizations and companies believe that a university's quality is measured by its position on the ranking tables like THE (Times Higher Education). The academic production of UMC researchers abroad (2 American and one from the United Kingdom) was evaluated by scientometric tools, such as Google Scholar, and production registered at the institution. In the case of the Brazilian UMC, the criterion was an analysis of the Lattes Curriculum and crossing with Qualis (Brazilian journal evaluation system). In addition to production, the degree and teaching time were analyzed. The data show that there is an influence of the teacher's degree in their production indexes (quantity and quality) and that teachers with more seniority have a more significant impact. Similar situations were found 
in Purdue, Manchester, and at the Federal University of São Paulo UNIFESP. The results of titration and production are different in Stanford, justifying its better classification in the ranking.

Keywords: World Class University, Undergraduate in Accounting, Faculty Profile, Evaluation of Scientific Production, Business Schools.

\section{INTRODUÇÃO}

Com sua origem na antiguidade, a ciência contábil se desenvolveu como ciência social aplicada pari passu ao desenvolvimento da sociedade e sua necessidade de registro, gerenciamento, normatização e regulamentação de seus bens patrimoniais.

Destaca-se o avanço teórico de pesquisas e construções do conhecimento na área para o desenvolvimento da contabilidade na forma atualmente definida por Sá (1999, p.42) como "ciência que estuda os fenômenos patrimoniais, preocupando-se com realidades, evidências e comportamentos dos mesmos, em relação a eficácia funcional das células sociais".

Considerando que a quase totalidade das pesquisas nas Ciências Contábeis ocorre em ambientes universitários e neste é que são absorvidas (por alunos em cursos de formação), em "Desafios e perspectivas da educação superior brasileira para a próxima década, 2011-2020" (United Nations Educational, Scientific and Cultural Organization UNESCO, 2012), foi colocando como papel dos docentes das universidades a capacidade de articular a pesquisa ao ensino no processo de aprendizagem.

Segundo Usoh, Ratu, Manongko, Taroreh e Preston (2018), a competitividade na economia internacional do conhecimento está intimamente ligada às universidades de excelência, responsáveis pela formação de recursos humanos tanto no ensino superior quanto na pós-graduação.

Desde a década de 1990 a comunidade acadêmica tem sido estimulada intencionalmente para o fenômeno "Universidade de Classe Mundial" (classificadas em ranquings comparativos) e, assim constar entre as melhores universidades para aumentar sua capacidade de competir internacionalmente com as universidades mais intensivas em 
pesquisa do mundo por recursos financeiros; atração, retenção e produção de talentos e reputação (Fu, Baker \& Zhang, 2020).

Desta forma, para contribuir com a discussão sobre a percepção de qualidade no ensino superior de Universidades consideradas de excelência, o presente estudo pretende avaliar a qualificação profissional, o tempo no magistério e a produção acadêmica de docentes de quatro Universidades de Classe Mundial: uma brasileira, duas americanas e uma do Reino Unido, que lecionam em cursos de formação para atuar em Ciências Contábeis (Accounting).

\section{REFERENCIAL TEÓRICO}

\subsection{A Indução de UMC no Mundo: rankings}

Os rankings universitários são ferramentas que surgem como consequência da globalização de mercados a fim de comparar a performance das Instituições de Ensino Superior (IES). Entretanto, como aponta Marginson (2014) parte dos esforços para se tornar uma Universidade de Classe Mundial (UMC) ou World Class University (WCU em inglês) são ligados ao desejo de estima tanto do corpo docente, que deseja ser associado a uma instituição de renome, quanto para o corpo discente que deseja estudar em uma instituição mundialmente reconhecida.

Desde o início dos anos 2000, a percepção sobre a qualidade desta formação começou a ser avaliada por inspeção e acreditação. Neste contexto, esta qualidade pode ser refletida em posições mais altas em rankings de classificação e, portanto, reconhecimento público de excelência do ensino e pesquisa (Ordorika \& Lloyd, 2015).

Com relação às metodologias para classificação de universidades, dentre as mais aceitas, podem ser mencionadas o Ranking Acadêmico de Universidades do Mundo (ARWU), que é conduzido pela Shanghai Jiao Tong University [http://www.shanghairanking.com]; o $\quad$ QS World University Ranking. [http://www.topuniversities.com] e o Times Higher Education (THE) World University Rankings [http://www. timeshighereducation.co.uk] que será adotado no presente estudo.

A coleta e processamento das informações de uma instituição para sua posterior classificação sob o prisma do THE considera as dimensões: ensino (o ambiente de 
aprendizagem); pesquisa (volume, receita de financiamento e reputação), perspectiva internacional (equipe, alunos e pesquisa); citações (influência da pesquisa); renda obtida da indústria (transferência de conhecimento). Todos os dados são auditados de forma independente pela PricewaterhouseCoopers $(\mathrm{PwC})$, tornando o THE World University Rankings o único a ser submetido a um escrutínio completo e independente desta natureza (https://www.timeshighereducation.com/world-university-rankings).

Muitas organizações e empresas medem a qualidade de uma universidade por sua posição nas tabelas de classificação. As universidades superiores nas listas de classificação são reconhecidas como sendo de maior qualidade. Esta é a razão de a classificação ser considerada um meio de validar as instituições de ensino superior para o mercado profissional (Marginson \& Van Der Wende, 2007).

Depreende-se então que o status de UMC implica em possuir certas características essenciais: alta concentração de talentos, recursos suficientes e políticas projetadas para estimular a busca pela excelência acadêmica e educacional. Para que uma universidade se torne de classe mundial deve esforçar-se pela excelência. Isso tem implicações óbvias para a qualidade do ensino superior (Chaimovich, 2014).

\subsection{A indução de UMC no Brasil: Política Pública}

A Coordenação de Aperfeiçoamento de Pessoal de Nível Superior- CAPES possui papel fundamental em várias ações governamentais de políticas públicas, dentre elas, podese destacar o lançamento do Edital 41/2017- PrInt (https://www.gov.br/capes/ptbr/centrais-de-conteudo/10112017Edital412017InternacionalizacaoPrInt2.pdf) onde foram selecionados 36 Projetos Institucionais de Internacionalização de Instituições de Ensino Superior (IES) ou de Institutos de Pesquisa (IP) que tenham Programas de PósGraduação (PPGs) recomendados pela CAPES no âmbito da última avaliação Quadrienal (2017).

Desta forma, foram elegíveis ao presente edital instituições que possuíam um Plano Institucional de Internacionalização ou documento congênere, com vigência que abranja o período do Projeto proposto (2018-2022). Dentre estas, está a Universidade Federal de São Paulo - UNIFESP que será objeto da análise deste estudo. 


\subsection{A importância da Titulação, Experiência e Produção acadêmica}

A pesquisa de Cordeiro, Lievorne e Pagani (2020) destaca a soberania das universidades americanas e do Reino Unido em ensino e pesquisa, refletida na sua excepcional classificação em ranquings universitários como o THE.

Considerando a titulação e sua influência nas competências do docente, pontuaram Vasconcelos, Cavalcante e Do Monte (2012, p.94) “Os cursos de doutorado podem influenciar nos conhecimentos didático-pedagógicos dos professores que o cursaram, melhorando o trabalho enquanto docentes."

Isaia e Bolzan (2010) destacam que os processos de "construção docente estão imbricados na atividade de aprender a ser professor" (p.5). Desta forma depreende-se que o tempo no exercício do magistério superior permite um aprimoramento das competências didáticas e melhoria no ensino-aprendizagem. Já para Arroyo (2000), a profissão docente é construída artesanalmente, a partir de práticas concretas, ou seja, tornamo-nos professores no exercício cotidiano da docência.

Cunha (2010) considera que nos processos de avaliação externa, vigentes para a universidade brasileira, os elementos que constituem indicadores de sucesso da atividade universitária são: o componente da docência e o componente da investigação.

A avaliação do número de trabalhos publicados, inicialmente amplamente aceita e utilizada tanto no Brasil como no exterior, hoje é insuficiente como forma de avaliação da contribuição científica do pesquisador. Apesar de críticas de alguns membros da comunidade acadêmica, a qualidade das publicações, ou seja, o interesse despertado pelo trabalho ou linha de pesquisa dentro da comunidade científica ganha destaque, fator esse que reflete no número de citações feitas a um determinado trabalho em função de sua relevância. (Thomaz, Assad \& Moreira, 2011).

Como reflexo da mudança na divulgação do resultado de pesquisas com a ampliação do uso de Tecnologias da Informação e Comunicação (TIC), ocorreu um crescimento significativo de repositórios dos diferentes canais de comunicação científica, informais e formais, abertos e restritos, especialmente em bases de dados. Hjørland (2002) destaca os estudos bibliométricos, principalmente com análises baseadas nas citações recebidas pela produção científica de uma comunidade acadêmica. 
Uma medida amplamente utilizada para medir o impacto científico são as citações, a previsão do número obtido por qualquer publicação representado pelo número de vezes em que o resultado da pesquisa (autor) é referenciado em outras produções

Com o objetivo de estimar o impacto acumulado da produção científica de um pesquisador, Hirsch (2005) desenvolveu um índice bibliométrico que considera aspectos relativos à qualidade (impacto) e à quantidade (número de artigos produzidos), o chamado índice $\mathrm{h}$. Sendo o índice $\mathrm{h}=$ número de artigos $(\mathrm{h})$ com número de citação $\geq \mathrm{h}$, um cientista com um h-Index de 37 tem 37 artigos citados pelo menos 37 vezes, por exemplo. O Web of Science é uma das bases de usa o h-Index, com vários bancos de dados e, atualmente mantido pela Clarivate Analytics (maiores detalhes no sito da CAPES: http://wwwperiodicos-capes-gov

.br.ezl.periodicos.capes.gov.br/index.php?option=com_pcollection\&mn=70\&smn=79\&ci $\mathrm{d}=81 \&$ ). As principais ferramentas para medir o índice h: Web of Science (pago) e Google Scholar (gratuito).

A avaliação do impacto dos artigos científicos publicados tem direta relação com a qualificação da produção acadêmica, especialmente em instituições de pesquisa e ensino superior e fazem parte dos indicadores que qualificam a excelência de uma UMC contribuindo para sua melhor posição no THE.

\section{METODOLOGIA}

Neste item são abordados os procedimentos metodológicos de pesquisa adotados neste trabalho. Em vista do contexto e dos objetivos estabelecidos, as metodologias adotadas foram de análise comparativa, quantitativa e qualitativa, utilizando pesquisa telematizada.

A pesquisa quanti-qualitativa possibilita considerar dados quantitativos e, ao mesmo tempo, valorar dados de maneira pormenorizada levando em consideração uma discussão particularizada, que abarca nuances específicas de uma dada realidade (Minayo, 1996), no caso, a produção docente nas UMC. 


\subsection{Base de dados para pesquisa}

A Universidade brasileira avaliada neste estudo é a Universidade Federal de São Paulo (UNIFESP), beneficiária do Edital 41/2017 por ser considerada com maior potencial de ampliar sua internacionalização institucional por consecução dos objetivos descritos no Plano de Desenvolvimento Institucional (PDI), com posição entre 601-800 na classificação geral do World University Rankings do THE de 2021, entre 101-200 no Impact Rankings de 2020 e na posição 48 do World Reputation Rankings de 2020.S College Rankings de 2021 e a $5^{\text {a }}$ no Latin America Rankings 2020.

Em paralelo, foram avaliadas três instituições estrangeiras da categoria UMC no THE. A escolha da Universidade de Stanford ocorreu pelo fato desta ocupar o $1^{\circ}$ lugar na área de Negócios e Economia na relação do THE e ter um resultado em citações excepcional: 99,9/100 (resultado da relevante produção acadêmica de docentes e discentes). Adicionalmente, no THE, Stanford University ocupa a $2^{\text {a }}$ posição no World University Rankings de 2021, a $4^{\text {a }}$ no US College Rankings de 2021 e a $3^{\text {a }}$ no World Reputation Rankings de 2020.

Em muitos países não é comum a oferta de Bacharelado em Ciências Contábeis, sendo normalmente ofertado como complemento curricular na formação de graduação em negócios. Para obter o título de bacharel, Bachelor of Science (BS) in Accounting é necessário que a Universidade ofereça essa modalidade (major).

Apesar de não ter um curso de Bacharelado em Ciências Contábeis, a Universidade de Stanford é considerada a principal na formação de recursos humanos na área de Negócios nos Estados Unidos (Graduate School of Business), desta forma, matrículas nas disciplinas do Departamento de Ciências Contábeis, ao longo de cursos de graduação em negócios têm por objetivo preparar o egresso para sua carreira em contabilidade ou para continuar seus estudos na Pós-graduação ofertada pela Escola de Negócios da instituição.

Para completar a análise proposta, investigou-se a formação (titulação) e a produção acadêmica dos docentes duas outras que oferecem o Bacharelado em Ciências Contábeis, dentro de escolas de negócios renomadas, que estão entre as 100 melhores do mundo em 2021 e com reputações semelhantes na classificação geral, uma nos Estados Unidos, a Univesidade de Purdue (Krannert School of Management) e outra no Reino Unido, a 
Universidade de Manchester (Alliance Manchester Business School), detalhes obtidos do THE a seguir.

- Purdue University West Lafayette, a número 94 na classificação geral do World University Rankings de 2021, a 47 no US College Rankings de 2021 e na posição 61 do World Reputation Rankings de 2020.

- University of Manchester, a número 51 na classificação geral do World University Rankings de 2021, a $8^{\text {a }}$ no Impact Rankings de 2020 e na posição 48 do World Reputation Rankings de 2020.

\subsection{Coleta de Dados}

Em cada uma das UMC foi avaliada a qualificação dos docentes para o ensino e pesquisa (titulação e carreira no magistério) bem como a pesquisa publicada em periódicos nos últimos anos disponível em bases de dados públicas, conforme descrição abaixo.

- Do Brasil

No caso Universidade Federal de São Paulo, utilizou-se as informações disponíveis na página institucional do Campus Osasco da Universidade Federal de São Paulo (https://www.unifesp.br/campus/osa2/) para construção da base de docentes com vínculo institucional e participantes do escopo do trabalho. Desta relação, foram consultadas as páginas pessoais dos docentes do Departamento de Ciências Contábeis que participam do programa de graduação dentro da Plataforma Lattes do Conselho Nacional de Desenvolvimento Científico e Tecnológico- CNPq para filtragem da produção científica veiculada em periódicos científicos de 2013 a 2016 e o registro da data da maior titulação (mestrado ou doutorado) para avaliar sua situação na carreira de forma comparativa as outras UMC.

Uma vez identificada a produção para cada docente, esta foi qualificada na Plataforma Sucupira mantida pela Coordenação de Aperfeiçoamento de Pessoal de Nível $\begin{array}{lllll}\text { Superior - } & \text { CAPES (Qualis Periódico - } & \text { CAPES) }\end{array}$ https://sucupira.capes.gov.br/sucupira/public/index.xhtml com a finalidade de promover o encontro relacionado a produção filtrada e a classificação de periódicos do último quadriênio disponível (2013-2016). Os periódicos avaliados de acordo com os dados para a área 27 (Administração Pública e de Empresas, Ciências Contábeis e Turismo). A 
ausência da avaliação quadrienal em 2020, em função da COVID-19, implicou nesta solução metodológica.

- Do exterior

No caso das universidades estrangeiras, foram consultadas as páginas pessoais dos docentes do Departamento de Ciências Contábeis envolvidos na formação de alunos na etapa de Graduação na UMC e registrado o número de artigos publicados pelo pesquisador desde 2016. Caso não haja registro ou as publicações extrapolam 2016 será atribuído o valor "0" (ausente).

Dentro das ferramentas gratuitas para avaliar a produção acadêmica, está o índice 'i10', criado pelo Google Scholar e usado no recurso Minhas citações do Google (https://guides.library.cornell.edu/impact/author-impact-10). i10-Index $=$ número de publicações com pelo menos 10 citações.

De forma paralela, foi realizada busca ativa nos perfis do Google Scholar utilizando o nome destes que apresenta o número de citação do Google Scholar, e os índices h-index e i10 index a partir de 2016, por não ser possível escolher outra data de início (https://scholar.google.com.br/citations?user=Sw1uAN4AAAAJ\&hl=ptBR\&authuser=1.)

\section{ANÁLISE E DISCUSSÕES}

O presente estudo visa analisar as características, a titulação, experiência e produção acadêmica dos últimos anos de três UMC, os resultados serão apresentados por instituição.

\subsection{Caracterização da Carreira Universitária nas UCM}

As informações sobre a estrutura da Carreira no Magistério Superior das Universidades Federais podem ser consultadas na página institucional da Unifesp (https://unifesp.br/reitoria/cppd/78-cppd/72-plano-de-carreiras-e-cargos-de-magisteriofederal). Entretanto para fins de análise, serão adotados critérios "ajustados" considerando 
o número de anos após a defesa do Doutorado para definir a posição na carreira do docente: inicial, intermediário, avançado e aposentado (acima de 65 anos).

Nos Estados Unidos, a carreira nas Universidades apresenta, de forma simplificada (https://thebestschools.org/magazine/professors-ranks/), as seguintes situações: Professor assistente; Professor associado; Professor (Full ou Titular em português) e Emérito (professor titular que se aposenta, mas continua a lecionar e a conduzir pesquisas no departamento).

O sistema de carreira universitária no Reino Unido (https://academicpositions.com/career-advice/uk-academic-job-titles-explained) se aproxima da realidade americana mostrada anteriormente neste texto. Apenas a nomenclatura apresenta variações, onde o chamado 'Leitor' (tradução livre de Lecturer) é o professor universitário (ensino e pesquisa). A saber: Assistente ou Associado (Assistant or Associate Lecturer); Professor universitário (Lecturer - análogo a professor assistente nos EU); Professor universitário sênior (Senior Lecturer - corresponde a professor associado nos EU); Professor titular (Professor); Honorário: é o análogo a professor titular de uma cadeira (Chair holder) e Emérito (aposentado após completar sessenta anos e com opção de continuar ativo no ensino e pesquisa).

Para fins de comparação com Stanford e Purdue, será adotada para a qualificação dos docentes do curso de graduação em Ciências Contábeis de Manchester (Reino Unido) a nomenclatura adaptada para a realidade americana.

\subsection{Descrição das UMC e docentes na oferta de formação: Graduação em Ciências Contábeis}

a) Universidade Federal de São Paulo (Brasil https://www.unifesp.br/campus/osa2/)

Fundada em 1933 através de recursos privados e doações governamentais, a universidade foi federalizada em 1956 com a lei n. ${ }^{\circ} 8.957$ de 1994, onde a então Escola Paulista de Medicina foi transformada em Universidade Federal de São Paulo - UNIFESP. O Bacharelado em Ciências Contábeis, ofertado na cidade de Osasco pela Escola Paulista de Política Economia e Negócios - EPPEN, campus que iniciou suas atividades no ano de 2011 e hoje possui o programa de pós-graduação stricto sensu, o Mestrado Acadêmico em Economia e Desenvolvimento. 


\section{- Titulação e Experiência do Corpo Docente}

Na UNIFESP, os docentes responsáveis pelas disciplinas em Ciências Contábeis (Accounting) têm um perfil heterogêneo quanto a sua posição na carreira. A maioria possui o título de doutor e vínculo institucional (tabela 1).

Tabela 1. Nível de formação máxima (titulação), cargo exercido na instituição e nível na carreira docente dos docentes responsáveis por disciplinas de formação em Ciências Contábeis na Universidade Federal de São Paulo (Brasil).

\section{Docente Titulação Ano da última titulação Cargo Nível na carreira}

\begin{tabular}{lllll}
\hline UN01 & Mestre & 2013 & Associado & Intermediário \\
UN02 & Doutor & 2005 & Titular & Avançado \\
UN03 & Doutor & 2004 & Titular & Avançado \\
UN04 & Doutor & 2005 & Titular & Avançado \\
UN05 & Mestre & 2013 & Associado & Intermediário \\
UN06 & Doutor & 2000 & Titular & Avançado \\
UN07 & Doutor & 2000 & Titular & Avançado \\
UN08 & Doutor & 2015 & Associado & Avançado \\
UN09 & Doutor & 2018 & Assistente & Inicial \\
UN10 & Doutor & 2011 & Associado & Avançado \\
UN11 & Doutor & 2019 & Assistente & Inicial \\
UN12 & Doutor & 1996 & Emérito & Aposentado \\
UN13 & Doutor & 1994 & Emérito & Aposentado \\
UN14 & Doutor & 2018 & Assistente & Inicial \\
UN15 & Doutor & 2015 & Associado & Avançado \\
UN16 & Doutor & 2010 & Associado & Avançado
\end{tabular}

Fonte: Autoria própria a partir de https://www.unifesp.br/campus/osa2/ e http://lattes.cnpq.br/

A tabela 1 destaca um corpo docente com bastante tempo de titulação, e experiência em ensino (dados obtidos do Lattes) e pesquisa, 68\% nas posições de titular e emérito. Destaca-se que os professores eméritos (UN12 e UN13) são aposentados em outra instituição e na Unifesp fazem parte do quadro regular de ensino e pesquisa. Somente 18,7\% em uma fase intermediária (UN01 e UN05) e 12,5\% no início da carreira (UN09, UN11 e UN14). Também se destaca a alta qualificação, $87.5 \%$ doutores e o restante com mestrado (sem especialistas). 


\section{- Produção Cientifica em Periódicos}

A Tabela 2 apresenta o número de artigos publicados por docente em cada um dos anos que compõe o quadriênio de 2013-2016 no caso da Universidade Federal de São Paulo.

Tabela 2: Relação das publicações de docentes da Universidade Federal de São Paulo (Brasil) em periódicos relatadas no Currículo Lattes no período de 2013 a 2016.

\begin{tabular}{lrrrrr} 
Docente & $\mathbf{2 0 1 3}$ & $\mathbf{2 0 1 4}$ & $\mathbf{2 0 1 5}$ & $\mathbf{2 0 1 6}$ & Total \\
\hline UN01 & 0 & 0 & 0 & 3 & 3 \\
UN02 & 0 & 1 & 0 & 0 & 1 \\
UN03 & 0 & 1 & 1 & 0 & 2 \\
UN04 & 0 & 0 & 0 & 0 & 0 \\
UN05 & 0 & 3 & 0 & 0 & 3 \\
UN06 & 2 & 3 & 7 & 4 & 16 \\
UN07 & 1 & 2 & 6 & 5 & 14 \\
UN08 & 0 & 0 & 0 & 0 & 0 \\
UN09 & 0 & 0 & 0 & 1 & 1 \\
UN10 & 1 & 0 & 0 & 0 & 1 \\
UN11 & 0 & 1 & 0 & 3 & 4 \\
UN12 & 1 & 0 & 0 & 0 & 1 \\
UN13 & 0 & 0 & 0 & 0 & 0 \\
UN14 & 0 & 0 & 0 & 1 & 1 \\
UN15 & 0 & 2 & 1 & 1 & 4 \\
UN16 & 1 & 2 & 7 & 2 & 12 \\
\hline
\end{tabular}

Fonte: Dados da pesquisa

Foram publicados 63 artigos pelos docentes do departamento de ciências contábeis da Universidade Federal de São Paulo no quadriênio 2013-2016. Com relação a constância, 3 professores tiveram artigos publicados em todos os anos contemplados pelo quadriênio (UN06, UN07 e UN16)) e outros 3 não tiveram nenhuma publicação veiculada em periódico científico no mesmo período (UN04, UN08 e UN13).

\section{A Internacionalização da produção docente}

Embora não tenha relação com a proposta inicial de pesquisa, foi considerado conveniente mostrar o alcance internacional dos artigos de autoria dos docentes do departamento de ciências contábeis da Universidade Federal de São Paulo. A tabela 3 
mostra a relacionamento entre país do periódico no qual o artigo foi publicado e docente autor.

Tabela 3: Produção docente distribuída por pais de origem dos periódicos onde o artigo foi publicado.

\begin{tabular}{lccccccc} 
Docente & Argentina & Brasil & Chile & $\begin{array}{c}\text { Estados } \\
\text { Unidos }\end{array}$ & Espanha & Uruguai & $\begin{array}{c}\text { Total } \\
\text { Geral }\end{array}$ \\
UN01 & - & 1 & 2 & - & - & - & 3 \\
UN02 & - & 1 & - & - & - & - & 1 \\
UN03 & - & 2 & - & - & - & - & 2 \\
UN05 & - & 3 & - & - & - & - & 3 \\
UN06 & - & 15 & - & - & 1 & - & 16 \\
UN07 & 1 & 7 & - & 4 & - & - & 12 \\
UN09 & - & - & - & 1 & - & - & 1 \\
UN10 & - & 1 & - & - & - & - & 1 \\
UN11 & - & 6 & - & - & - & - & 6 \\
UN12 & - & 1 & - & - & - & - & 1 \\
UN14 & - & 1 & - & - & - & - & 1 \\
UN15 & - & 4 & - & - & - & - & 4 \\
UN16 & - & 9 & - & - & 2 & 1 & 12 \\
\hline
\end{tabular}

Fonte: Dados da pesquisa

A tabela 3 mostra que no Quadriênio 2013-2016, os docentes publicaram 12 artigos em periódicos internacionais, correspondentes a 19,05\% de toda a base. O docente com portfólio mais internacionalizado (UN07) concentra 5 publicações em periódicos internacionais abrangendo 2 países além do Brasil, Estados Unidos da América e Argentina, seguida do UN16 que possui 3 publicações internacionais na Espanha e Uruguai, tendo os demais docentes até 2 publicações em um país concentrado.

A produção internacional destes 2 docentes corresponde a $75 \%$ das publicações veiculados em periódicos internacionais, logo podemos dizer que há uma concentração neste quesito. Tal fenômeno pode ser explicado pelas áreas temáticas de pesquisa de cada um dos docentes.

\section{A Qualificação da produção docente de acordo com a CAPES}

$\mathrm{Na}$ última Quadrienal, a produção docente foi qualificada em oito estratos considerando os critérios definidos pela CAPES, sendo eles em ordem decrescente: A1, A2, B1, B2, B3, B4, B5 e C. Os periódicos que foram identificados nos Currículos Lattes 
dos docentes do Departamento de Ciências Contábeis da UNIFESP foram classificados conforme a área de Administração Pública e de Empresas, Ciências Contábeis e Turismo (tabela 4).

Tabela 4. Qualificação da Produção acadêmica dos professores do Bacharelado em Ciências Contábeis da Universidade Federal de São Paulo, de 2013 a 2016 conforme critérios Qualis/CAPES.

\begin{tabular}{ccccccccc} 
Docente & A1 & A2 & B1 & B2 & B3 & B4 & B5 & C \\
\hline UN01 & - & - & - & - & - & 3 & - & - \\
UN02 & - & - & - & - & - & 1 & - & - \\
UN03 & 1 & - & 1 & - & - & - & - & - \\
UN05 & - & - & - & - & - & - & - & - \\
UN06 & - & 1 & 3 & 3 & 2 & 2 & 1 & - \\
UN07 & - & - & - & 1 & 4 & 1 & - & - \\
UN09 & 1 & - & - & & - & - & - & - \\
UN10 & - & 1 & - & & - & - & - & - \\
UN11 & - & - & - & 1 & 1 & 2 & 1 & - \\
UN12 & - & - & - & - & - & - & - & - \\
UN14 & - & - & - & - & 1 & - & - & - \\
UN15 & - & - & - & - & 1 & 3 & - & - \\
UN16 & - & - & - & - & - & - & - & 1 \\
\hline Total & $\mathbf{2}$ & $\mathbf{2}$ & $\mathbf{4}$ & $\mathbf{5}$ & $\mathbf{9}$ & $\mathbf{1 2}$ & $\mathbf{2}$ & $\mathbf{1}$
\end{tabular}

Fonte: Dados da pesquisa.

Dos 63 artigos publicados pelo grupo (2013-2016), foram extraídos dos resultados o total de 26 artigos por não se enquadrarem nos critérios estabelecidos pela CAPES e seus oito estratos de classificação. Considerando a produção qualificada, podemos verificar (tabela 5) que a maioria dos artigos veiculados em periódicos científicos estão no estrato B4, correspondendo a 32,43\% da produção total que faz parte da base de pesquisa e onde os periódicos já foram estratificados pela Qualis - CAPES. As maiores contribuições neste estrato partiram dos docentes UN15 e UN01 com 3 artigos cada.

A segunda maior concentração de produção está no estrato B3, com 9 publicações representando $24,32 \%$ do total, onde a maior contribuição partiu da produção científica publicada em periódicos da UN07 com 4 artigos. Seguidos pelos estratos B1 e B2 que representam somados também $24,32 \%$ de toda a produção tendo o UN06 como responsável por maior parte da contribuição nos estratos, tendo 6 artigos. 
É importante observar que 4 artigos ocupam os estratos A1 e A2 conforme periódico em que foram publicados, e esta amostra é pulverizada, não tendo - no quadriênio- um docente com mais de 1 publicação nestes estratos.

b) Universidade de Stanford (Estados Unidos- https://www.stanford.edu/)

Localizada no coração do Vale do Silício, a Universidade de Stanford foi fundada em 1885 e é um dos maiores campi dos Estados Unidos, com sete escolas: Escola de Ciências da Terra, Energia e Meio Ambiente; Escola de Pós-Graduação em Educação; Escola de engenharia; Escola de Humanidades e Ciências; Escola de Direito; e Faculdade de Medicina e Escola de Negócios (Graduate School of Businesshttps://www.gsb.stanford.edu/) onde funciona a graduação em Ciências Contábeis (Accounting).

Stanford tem 19 ganhadores do Prêmio Nobel e vários ex-alunos de destaque. No total, as empresas fundadas por afiliados e ex-alunos de Stanford geram mais de US \$ 2,7 trilhões de receita anual. Essas empresas incluem Google, Nike, Netflix, Hewlett-Packard, Sun Microsystems, Instagram e Charles Schwab.

A Universidade é considerada o principal destino nos Estados Unidos para uma formação continuada no contexto da Contabilidade, na Stanford Graduate School of Business (https://www.gsb.stanford.edu). Dentre os docentes da Escola de Pós-graduação em Negócios de Stanford, foram selecionados neste estudo todos os professores que oferecem as disciplinas na trajetória formativa da subárea Accounting (https://www.gsb.stanford.edu/faculty-research/faculty/academic-areas/accounting).

\section{- Titulação e Experiência do Corpo Docente (Accounting)}

Na universidade de Stanford, os docentes responsáveis pelas disciplinas em Programas de formação em Ciências Contábeis (Accounting) têm um perfil heterogêneo quanto a sua posição na carreira. Todavia todos possuem o título de doutor e vínculo (tabela $5)$. 
Tabela 5. Nível de formação máxima (titulação), cargo exercido na instituição e nível na carreira docente dos docentes responsáveis por disciplinas de formação em Ciências Contábeis na Universidade de Stanford (Estados Unidos).

\begin{tabular}{llll} 
Docente & Titulação & Cargo & Nível na carreira \\
\hline SD01 & Doutor & Assistente & Inicial \\
SD02 & Doutor & Assistente & Inicial \\
SD03 & Doutor & Associado & Intermediário \\
SD04 & Doutor & Associado & Intermediário \\
SD05 & Doutor & Assistente & Inicial \\
SD06 & Doutor & Titular & Avançado \\
SD07 & Doutor & Assistente & Inicial \\
SD08 & Doutor & Titular & Avançado \\
SD09 & Doutor & Assistente & Inicial \\
SD10 & Doutor & Titular & Avançado \\
SD11 & Doutor & Emérito & Aposentado \\
SD12 & Doutor & Titular & Avançado \\
SD13 & Doutor & Associado & Intermediário \\
SD14 & Doutor & Emérito & Aposentado \\
SD15 & Doutor & Titular & Avançado \\
SD16 & Doutor & Associado & Intermediário \\
SD17 & Doutor & Emérito & Aposentado \\
SD18 & Doutor & Associado & Intermediário \\
& & & \\
& & & \\
And & &
\end{tabular}

Fonte: Dados obtidos de https://www.gsb.stanford.edu/faculty-research/faculty. Acesso jan 2021.

Destaca-se a presença de 3 professores eméritos no corpo docente, as outras categorias (assistente, associado e titular) têm o mesmo número de representantes: 5 de cada. Depreende-se da tabela 16 que a maioria do corpo docente tem bastante experiência em ensino (emérito, titular e associados).

\section{- Produção Cientifica em Periódicos: quantidade e qualidade (impacto)}

Dos 18 professores do grupo avaliado, cinco não apresentaram perfil no Google Scholar, e sua produção foi estimada a partir das informações que constam na sua página institucional. Desta forma, fundamentada simplesmente na coleta de dados disponibilizados pela instituição, a tabela 6 a seguir mostra a quantidade de publicações destes cinco docentes sem perfil de Google Scholar. 
Tabela 6. Relação das publicações em periódicos relatadas na página pessoal do professor no repositório institucional (Universidade de Stanford, EU) no período de 2016

\begin{tabular}{lccccccc} 
Docente & 2016 & 2017 & 2018 & 2019 & 2020 & 2021 & Total \\
\hline SD14 & 3 & 2 & 1 & 3 & 0 & 1 & 10 \\
SD15 & 1 & 0 & 0 & 0 & 0 & 0 & 1 \\
SD16 & 1 & 0 & 0 & 1 & 0 & 0 & 2 \\
SD17 & 1 & 4 & 2 & 1 & 2 & 0 & 10 \\
SD18 & 0 & 0 & 0 & 1 & 1 & 0 & 2 \\
\hline
\end{tabular}

Fonte: Dados obtidos de https://www.gsb.stanford.edu/faculty-research/faculty. Acesso jan 2021.

Deve-se considerar que o preenchimento do Perfil no Google Scholar é uma decisão pessoal e por já estarem aposentados pode não ter sido considerado necessário pelos pesquisadores SD14 e SD17. Desta forma, qualificar o impacto da produção destes seria inadequado sem o uso de cientometria. Todavia, observando o mesmo período avaliado pelo Google Scholar neste estudo (2016-2021), percebe-se pelas informações disponibilizadas na sua página institucional que publicam regularmente.

Contudo, os docentes SD15, SD16 e SD18 têm uma produção bem abaixo dos colegas de departamento e do esperado de uma UCM, inclusive pelo fato de SD15 ser um professor titular.

Destaca-se que no caso da Universidade de Stanford, a grande maioria dos docentes (72\%) pode ser avaliado por meio da cientometria. A Tabela 7 apresenta os pesquisadores da Universidade de Stanford, com seus respectivos, número de citações, índice-h e i10 da base Google Scholar do período 2016 até o momento. 
Tabela 7. Impacto da Produção acadêmica dos professores responsáveis pela oferta de cursos na formação em Ciências Contábeis da Universidade de Stanford (Estados Unidos), desde 2016. Docente Citações h-index i10 index

\begin{tabular}{lrrr}
\hline SD01 & 34 & 2 & 1 \\
SD02 & 79 & 4 & 2 \\
SD03 & 883 & 15 & 16 \\
SD04 & 477 & 8 & 8 \\
SD05 & 140 & 6 & 6 \\
SD06 & 3240 & 18 & 19 \\
SD07 & 36 & 3 & 2 \\
SD08 & 4642 & 17 & 26 \\
SD09 & 94 & 4 & 2 \\
SD10 & 8947 & 35 & 50 \\
SD11 & 63300 & 56 & 111 \\
SD12 & 6499 & 29 & 39 \\
SD13 & 619 & 14 & 17 \\
\hline
\end{tabular}

Fonte: página institucional docente com ligação ao Google Scholar.

O SD11, emérito, tem o maior número de citações e índices h e i10 do grupo, uma ordem de magnitude acima dos titulares (valores mais altos: SD10> SD12>SD08>SD06) e na sequência, uma ordem de magnitude menor três associados (SD03, SD04 e SD13)

c) Universidade de Purdue (Estados Unidos - https://www.purdue.edu/)

Ranqueada como a 5 ${ }^{\text {a }}$ Universidade mais inovativa nos Estados Unidos em 2021 a Purdue University, foi fundada em 1869 e tem diversos campi, incluindo o que está localizado na cidade de West Lafayette onde está a Escola de Negócios: Krannert School of Management, que oferta o Bacharelado em Ciências Contábeis.

Quatro cientistas da Universidade de Purdue foram Prêmio Nobel enquanto estavam na universidade. O Purdue Research Park, estabelecido em 1961, permite que pesquisadores experientes da universidade desenvolvam seu trabalho em colaboração com empresas privadas e indústrias de alta tecnologia.

- Titulação e Experiência do Corpo Docente em Purdue (BSc Accounting) 
$\mathrm{Na}$ universidade de Purdue, os docentes responsáveis pelas disciplinas do Bacharelado em Ciências Contábeis (Accounting) têm um perfil heterogêneo quanto a sua posição na carreira. A significativa maioria tem o título de doutor (tabela 8).

Tabela 8. Nível de formação máxima (titulação), cargo exercido na instituição e nível na carreira docente dos docentes responsáveis por disciplinas do Bacharelado em Ciências Contábeis na Universidade de Purdue (Estados Unidos).

\begin{tabular}{llll} 
Docente & Titulação & Cargo & Nível na carreira \\
\hline PD01 & Doutor & Titular & Avançado \\
PD02 & Doutor & Assistente & Inicial \\
PD03 & Doutor & Associado & Intermediário \\
PD04 & Doutor & Associado & Intermediário \\
PD05 & Doutor & Assistente & Inicial \\
PD06 & Doutor & Assistente & Inicial \\
PD07 & Doutor & Assistente & Inicial \\
PD08 & Doutor & Titular & Avançado \\
PD09 & Doutor & Titular & Avançado \\
PD10 & Doutor & Emérito & Aposentado \\
PD11 & Doutor & Emérito & Aposentado \\
PD12 & Especialista & Assistente & Inicial \\
PD13 & Mestre & Assistente & Inicial \\
PD14 & Doutor & Assistente & Inicial \\
PD15 & Doutor & Titular & Avançado \\
PD16 & Doutor & Assistente & Inicial \\
PD17 & Mestre & Assistente & Inicial \\
PD18 & Doutor & Assistente & Inicial \\
\hline & & & \\
\hline
\end{tabular}

Fonte: Dados obtidos de https://krannert.purdue.edu/directory/view.php?search=FacArea\&FacAreaList=51. Acesso jan 2021.

Mais da metade dos 18 docentes responsáveis pelas disciplinas do Bacharelado em Ciências Contábeis (Accounting) está no estágio inicial quanto a sua posição na carreira (55\%), $11 \%$ está em uma etapa intermediária (PD04 e PD04). O restante, cerca de um terço, é bastante experiente em ensino (titular ou emérito). Destaca-se a presença de um especialista (PD12) e um mestre (PD13) neste grupo formado majoritariamente (cerca de 90\%) por doutores (tabela 8).

Revista ENIAC Pesquisa, Guarulhos (SP), V.10, n.1, fev.- ago.2021 
- Produção Cientifica em Periódicos: quantidade e qualidade (impacto)

Dos 18 professores do grupo avaliado, seis não apresentaram perfil no Google Scholar, e sua produção foi estimada a partir das informações que constam na sua página institucional. Desta forma, fundamentada simplesmente na coleta de dados disponibilizados pela instituição na sua página pessoal na Krannert School of Management da Universidade de Purdue, a tabela 9 mostra a quantidade de publicações destes seis docentes sem perfil de Google Scholar.

Tabela 9. Relação das publicações em periódicos relatadas na página pessoal do professor no repositório institucional (Universidade de Purdue, Estados Unidos) no período de 2016 a fevereiro de 2021.

\begin{tabular}{lrrrrrrr} 
Docente & 2016 & 2017 & 2018 & 2019 & 2020 & 2021 & Total \\
\hline PD07 & 1 & 0 & 0 & 0 & 0 & 2 & 3 \\
PD08 & 0 & 0 & 1 & 4 & 2 & 0 & 7 \\
PD09 & 0 & 1 & 0 & 0 & 1 & 0 & 2 \\
PD10 & 0 & 0 & 0 & 0 & 0 & 0 & 0 \\
PD11 & 0 & 0 & 0 & 0 & 0 & 0 & 0 \\
PD12 & 0 & 0 & 0 & 0 & 0 & 0 & 0 \\
PD13 & 0 & 0 & 0 & 0 & 0 & 0 & 0 \\
PD14 & 0 & 0 & 0 & 0 & 0 & 0 & 0 \\
PD15 & 0 & 0 & 0 & 0 & 0 & 0 & 0 \\
PD16 & 0 & 0 & 0 & 0 & 0 & 0 & 0 \\
PD17 & 0 & 0 & 0 & 0 & 0 & 0 & 0 \\
PD18 & 0 & 0 & 0 & 0 & 0 & 0 & 0 \\
\hline
\end{tabular}

Fonte: Dados obtidos de https://krannert.purdue.edu/fast-tracks/faculty-staff.php. Acesso jan 2021.

A tabela 9 evidencia a ausência ou o reduzido número de publicações acadêmicas relatadas no sítio institucional de 2/3 dos docentes do Bacharelado em Ciências Contábeis da Universidade de Purdue independentemente de sua maturidade como professor universitário. Foi observado que em alguns casos, no seu perfil o pesquisador apresenta um arquivo em pdf com seu currículo vitae, mas geralmente destacando neste documento suas atividades profissionais na área de negócios e gestão. O docente PD08 é o único que se destaca neste grupo que não apresenta perfil cadastrado no Google Scholar, com 
publicações concentradas nos anos de 2018, 2019 e 2020 (como a pesquisa foi feita em janeiro, ainda não foi possível estimar 2021).

Uma minoria dos docentes $(33 \%)$ pode ser avaliada por meio da cientometria. Entretanto, diferente do caso de Stanford, as páginas pessoais não têm ligação com o Google Scholar, sendo assim os resultados mostrados a seguir foram obtidos por busca ativa no Google (perfis cadastrados). A Tabela 10 apresenta os pesquisadores da Universidade de Purdue, com seus respectivos, número de citações, índice-h e i10 da base Google Scholar do período 2016 até o momento.

Tabela 10. Impacto da Produção acadêmica dos professores responsáveis pela oferta de cursos no Bacharelado em Ciências Contábeis da Universidade de Purdue (Estados Unidos), desde 2016. Docente Citação h-index i10-index

\begin{tabular}{rrrr}
\hline PD01 & 94 & 1 & 1 \\
PD02 & 9 & 2 & 0 \\
PD03 & 464 & 9 & 9 \\
PD04 & 260 & 5 & 5 \\
PD05 & 2 & 1 & 0 \\
PD06 & 149 & 5 & 4 \\
\hline
\end{tabular}

Fonte: Dados obtidos por busca ativa pelo perfil do docente em https://scholar.google.com/. Acesso em jan 21.

Comparando as duas Universidades americanas, percebe-se uma diferença na produção bibliográfica do corpo docente significativamente menor em Purdue considerando a ferramenta Google Scholar considerando as citações, o h-index e o i10index.

d) Universidade de Manchester (Reino Unido https://www.manchester.ac.uk/discover/history-heritage/)

A Victoria University of Manchester se desenvolveu a partir do Owens College, fundado em 1851, que a partir de 1872 incorporou a Royal School of Medicine and Surgery. A Universidade de Manchester foi a alavanca da era nuclear, com Ernest Rutherford que provou à divisão do átomo. Além disso, a universidade também conta com outros 24 ganhadores do Prêmio Nobel entre seus funcionários e alunos atuais e antigos: 
Alliance Manchester Business School é a maior escola de negócios e gestão baseada em campus no Reino Unido. O Bacharelado em Ciências Contábeis, (https://www.alliancembs.manchester.ac.uk/study/undergraduate/bsc-hons-accounting/2 é descrito como profissionalmente orientado e, elaborado em conjunto com o Programa de Parceria de Graduação (UPP) do Instituto de Contadores Credenciados na Inglaterra e País de Gales (ICAEW).

- Titulação e Experiência do Corpo Docente em Manchester(Bsc Accounting)

Não foram identificados docentes com o perfil de Assistant ou Associate Lecturer neste estudo. Entretanto, percebe-se que o tempo de exercício e não a titulação permite assumir a posição de professor associado (MC04 e MC09) na Universidade de Manchester (tabela 11). Dezenove dos vinte e um professores possuem o título de doutor (90\%).

Tabela 11. Nível de formação máxima (titulação), cargo exercido na instituição e nível na carreira docente dos docentes responsáveis por disciplinas na graduação em Ciências Contábeis na Universidade de Manchester (Reino Unido). 


\begin{tabular}{llll} 
Docente & Titulação & Cargo & Nível na carreira \\
\hline MC01 & Doutor & Associado & Intermediário \\
MC02 & Doutor & Titular & Avançado \\
MC03 & Doutor & Associado & Intermediário \\
MC04 & Mestre & Associado & Intermediário \\
MC05 & Doutor & Assistente & Inicial \\
MC06 & Doutor & Associado & Intermediário \\
MC07 & Doutor & Assistente & Inicial \\
MC08 & Doutor & Assistente & Inicial \\
MC09 & Especialista & Associado & Intermediário \\
MC10 & Doutor & Associado & Intermediário \\
MC11 & Doutor & Assistente & Inicial \\
MC12 & Doutor & Honorário & Avançado \\
MC13 & Doutor & Titular & Avançado \\
MC14 & Doutor & Associado & Intermediário \\
MC15 & Doutor & Titular & Avançado \\
MC16 & Doutor & Emérito & Aposentado \\
MC17 & Doutor & Assistente & Inicial \\
MC18 & Doutor & Associado & Intermediário \\
MC19 & Doutor & Associado & Intermediário \\
MC20 & Doutor & Titular & Avançado \\
MC21 & Doutor & Titular & Avançado \\
\hline
\end{tabular}

Fonte: Dados obtidos de https://www.research.manchester.ac.uk/portal/en/researchers/search.html. Acesso jan 2021.

Os dados institucionais para o corpo docente indicam que dos 21 professores que ministram as disciplinas no curso de graduação em Ciências contábeis, a maioria é bastante experiente. No estágio avançado estão 9 docentes na categoria de titulares e um na condição de Emérito (MC16) e no intermediário os 6 associados. Desta forma, apenas 5 iniciando a carreira (assistentes: MC5, MC7, MC8, MC11 e MC17) em ensino, cerca de 25\% (tabela $11)$.

- Produção Cientifica em Periódicos: quantidade e qualidade (impacto)

Dos 21 professores do bacharelado em contabilidade, a maioria (quinze) não apresenta um perfil no Google Scholar, e sua produção foi avaliada a partir das informações que constam na sua página institucional. A partir da coleta de dados, a tabela 12 revela a Revista ENIAC Pesquisa, Guarulhos (SP), V.10, n.1, fev.- ago.2021 
quantidade de publicações destes docentes sem perfil de Google Scholar, o que corresponde a $71 \%$.

Tabela 12. Relação das publicações em periódicos relatadas na página pessoal do professor no repositório institucional (Universidade de Manchester, RU) no período de 2016 a fevereiro de

2021.

\begin{tabular}{lccccccc} 
Docente & 2016 & 2017 & 2018 & 2019 & 2020 & 2021 & Total \\
\hline MC01 & 1 & 0 & 1 & 0 & 1 & 0 & 3 \\
MC02 & 0 & 6 & 1 & 3 & 5 & 0 & 15 \\
MC03 & 1 & 1 & 1 & 2 & 1 & 0 & 6 \\
MC04 & 0 & 0 & 0 & 0 & 0 & 0 & 0 \\
MC05 & 0 & 0 & 1 & 1 & 0 & 1 & 3 \\
MC06 & 0 & 0 & 0 & 0 & 0 & 0 & 0 \\
MC07 & 0 & 1 & 0 & 2 & 1 & 1 & 5 \\
MC08 & 1 & 0 & 2 & 0 & 2 & 0 & 5 \\
MC09 & 0 & 0 & 0 & 0 & 0 & 0 & 0 \\
MC10 & 4 & 8 & 4 & 3 & 1 & 0 & 20 \\
MC11 & 1 & 0 & 0 & 0 & 0 & 0 & 1 \\
MC12 & 2 & 1 & 1 & 3 & 0 & 1 & 8 \\
MC13 & 3 & 3 & 2 & 1 & 1 & 1 & 11 \\
MC14 & 0 & 0 & 2 & 0 & 0 & 0 & 2 \\
MC15 & 7 & 0 & 0 & 2 & 1 & 0 & 10
\end{tabular}

Fonte: Dados obtidos de https://www.research.manchester.ac.uk/portal/en/researchers/search.html. Acesso jan 2021.

A análise da Tabela 12 mostra que neste grupo, os iniciantes (assistentes) produziram poucos artigos no período avaliado (MC05, MC07, MC08 e MC11) e que os dois docentes sem doutorado (MC04 e MC09) não apresentam publicações em suas páginas institucionais.

Dos docentes sem Google Scholar, o pesquisador MC10 (associado) se destaca com grande produção no ano de 2018. Os titulares apresentam regularidade na produção (entre 8 e 15 artigos publicados em periódicos no período).

No caso da Universidade de Manchester, a minoria dos docentes (28\%) preencheu seu perfil no Google Scholar e sua produção acadêmica foi avaliada por meio da cientometria. A Tabela 13 a seguir apresenta os pesquisadores desta insituição com seus Revista ENIAC Pesquisa, Guarulhos (SP), V.10, n.1, fev.- ago.2021 
respectivos, número de citações, índice-h e i10 da base Google Scholar do período 2016 até o momento.

Tabela 13. Impacto da Produção acadêmica dos professores responsáveis pela oferta de cursos na graduação do Bacharelado em Ciências Contábeis da Universidade de Manchester (Reino Unido), desde 2016.

\begin{tabular}{lrrr} 
Docente & Citações & h-index & i10- index \\
\hline MC16 & 941 & 15 & 25 \\
MC17 & 117 & 4 & 4 \\
MC18 & 1545 & 10 & 10 \\
MC19 & 2975 & 19 & 28 \\
MC20 & 456 & 11 & 12 \\
MC21 & 766 & 14 & 18 \\
\hline
\end{tabular}

Fonte: página institucional docente com ligação ao Google Scholar.

Os valores de Manchester diferem muito dos observados na Tabela 7 para docentes em Stanford. Observa-se que os professores com maior impacto estão em uma fase intermediária da sua carreira (MC18 e MC19) e o docente MC17 tem alto impacto acadêmico considerando seus pares, mesmo estando na fase inicial da sua carreira (assistente). O professor emérito MC16 tem impacto semelhante ao associado SD03 na Universidade americana, mas não se aproxima do apresentado por SD11 (citações: 63.300; h-index: 56; i10-index: 111). Entretanto, não difere muito da realidade na Universidade de Purdue (tabela 10).

Finalmente, as análises também mostraram que as universidades do eixo Estados Unidos e Reino Unido presentes no ranking, são instituições semi-privadas ou privadas, que captam recursos de diversos meios além dos repasses governamentais, permitindo uma maior autonomia financeira e de alocação de recursos para as instituições (Dobbins, Knill \& Vogtle, 2019) diferente de uma Universidade Federal.

\section{CONSIDERAÇÕES FINAIS}

As universidades estadunidenses e inglesas, por possuírem renome internacional, geralmente se distribuem em primeiras colocações dos rankings, pois seu status lhes garante a captação de talentos tanto nacionais, quanto internacionais e por isso foram objeto 
do presente estudo. Por outro lado, a Política para a Internacionalização das instituições no Brasil começa em 2017 com a escolha de 36 instituições, dentre elas a UNIFESP, também avaliada nos ranquings internacionais como THE.

A titulação está geralmente associada à maior produção acadêmica nesta amostra, onde especialistas e mestres tem menos artigos publicados, entretanto, a fase avançada na carreira nem sempre significa maior produção de artigos publicados em periódicos.

Os resultados mostram uma produção semelhante em números entre UNIFESP, Purdue, Manchester e mesmo Stanford, que se destaca pelo uso do Google Scholar para um grupo de seus docentes com altíssimo impacto no h-index e citações.

No caso da UNIFESP, no quadriênio de 2013-2016, sob a ótica de internacionalização, 19,05\% da produção docente ocorreu em periódicos da Argentina, Chile, Estados Unidos da América, Uruguai e Espanha. Adicionalmente, a maior concentração ocupou os estratos B3 e B4 considerados de média relevância.

Em todas as UMCs avaliadas uma parcela dos docentes que não teve artigos publicados em periódicos científicos evidenciado pela metodologia utilizada.

Adotado por ser uma base de consulta gratuita, o Google Scholar também não é totalmente sem restrições, seu preenchimento é voluntário e ainda não é unânime dentre os pesquisadores.

Além disso, apesar das vantagens do h-index na avaliação do impacto da produção, pois, seu uso permite comparações diretas dentro das disciplinas/ área de conhecimento e estima a quantidade e o impacto por um único valor, este apresenta limitações no caso de pesquisadores no início de carreira e que não tenham artigos indexados na Web of Science.

$\mathrm{O}$ estudo mostra que a produção acadêmica dos docentes vinculados as instituições estrangeiras, Universidade de Stanford (EU), Universidade de Purdue e Universidade de Manchester (RU) refletem as suas posições em rankings de classificação para Universidades de Classe Mundial considerando a análise cientométrica.

Considerando as informações avaliadas nesta pesquisa, o critério de excelência da pesquisa que coloca Stanford como segunda e Manchester como $51^{\mathrm{a}}$ e Purdue como $94^{\mathrm{a}}$ no THE em 2021 é coerente, mesmo com o desafio de se utilizar unicamente uma ferramenta gratuita como o Google Scholar.

Como sugestão para futuros trabalhos poderiam ser avaliadas as questões de "reconhecimento internacional" das instituições ranqueadas no THE, a fim de correlacionar os resultados com marketing e networking destas IES.

Revista ENIAC Pesquisa, Guarulhos (SP), V.10, n.1, fev.- ago.2021 


\section{REFERÊNCIAS}

Arroyo, M.G. (2000). Ofício de mestre: imagens e auto-imagens. Petrópolis: Vozes.

Chaimovich, H. (janeiro, 2014). Brazil, World-Class universities and excellence in research. Anais do Symposium ABC-FAPESP: Excellence In Higher Education. São Paulo, SP.

Cordeiro, L.G., Lievore, C., \& Pagani, R.N. (2020). Instituições de Ensino Superior no ranking Times Higher Education: uma análise sobre ensino, pesquisa e renda industrial. Revista Stricto sensu, Ponta Grossa, 5(1), 43-58, jun.

Cunha, M.I. (2010). Trajetórias e lugares de formação da docência universitária: da perspectiva individual ao espaço institucional. Araraquara: Junqueira \& Marin Editores, 2010.

Dobbins, M., Knill, C., \& Vögtle, E.M. (2011). An analytical framework for the cross-country comparison of higher education governance. Higher education, 62(5), 665-683.

Fu, Y.C., Baker, D.P., \& Zhang, L. (2020). Engineering a World Class University? The Impact of Taiwan's World Class University Project on Scientific Productivity. High Educ Policy, 33, 555-570.

Hirsch, J.E. (2005). An index to quantify an individual's scientific research output. PNAS USA, Washington, 102(46), 16569-16572, Nov.

Hjörland, B. (2002). Domain analysis in information science: eleven approaches traditional as well as innovative. Journal of Documentation, London, 58(4), 422- 462.

Isaia, S.M.A., \& Bolzan, D.P.V. (abril, 2010). Movimentos construtivos da docência/aprendizagem: tessituras formativas. Anais do XV Encontro Nacional de Didática e Prática de Ensino, ENDIPE. Belo Horizonte. p. 114.

Marginson, S. (2014). University rankings and social science. European Journal of Education, 49(1), 45-59.

Marginson, S., \& Van Der Wende, M. (2007). To rank or to be ranked: the impact of global rankings in higher education. Journal of Studies in International Education, 11(3-4), 1-24.

Minayo, M.C.S. (1996). O Desafio do Conhecimento: pesquisa qualitativa em saúde (4a ed). São Paulo: HUCITEC - ABRASCO.

Ordorika, I., \& Lloyd, M. (2015) International rankings and the contest for university hegemony. Journal of Education Policy, 30(3), 385-405.

Sá, A.L. de (1999). Teoria da Contabilidade (2. ed). São Paulo: Editora Atlas.

Thomaz, P.G., Assad, R.S., \& Moreira, L.F.P. (2011). Uso do Fator de impacto e do índice H para avaliar pesquisadores e publicações. Arq. Bras. Cardiol., São Paulo, 96(2), 90-93, Feb.

United Nations Educational, Scientific and Cultural Organization [UNESCO] (2012). Desafios e perspectivas da educação superior brasileira para a próxima década. Paulo Speller, Fabiane Robl e Stela Maria Meneghel (org.). Brasília: UNESCO, CNE, MEC, 2012. 
Usoh, E.J., Ratu, D., Manongko, A., Taroreh, J., \& Preston, G. (2018). Strategic Planning towards a World-Class University. ICIEVE 2017 IOP Publishing IOP Conf. Series: Materials Science and Engineering, 306, 012035.

Vasconcelos, A.F., Cavalcante, P.R.N., \& Do Monte, P. A. (2012). Fatores que influenciam as competências em docentes de Ciências Contábeis. Veredas FAVIP (Online), 5(1-2), 86101. Recuperado em 10 fevereiro 2021, de http://veredas.favip.edu.br/ojs/index.php/veredas1/article/view/8 